\title{
Editorial
}

\section{New Highlights in Gravitationally Lensed Quasar Research}

Just thirty years after the discovery of the first gravitationally lensed quasar is a good opportunity to summarize some recent advances in the field, and to attract the attention of students and senior astronomers about its potential. Once the standard scenario has been tested on more than 100 gravitationally lensed quasars: several images of a background quasar are produced by an intervening massive galaxy (or association of galaxies), several current efforts focus on the exploration of new kinds of lenses (apart from galaxies), the composition and structure of lensing galaxies, the nature and structure of lensed quasars, and the involved cosmological model.

This special issue includes a review of cosmic strings and their gravitational lens effects by one of the most prominent groups worldwide studying this kind of cosmic objects. Although there are no confirmed cosmic strings and their gravitational lensing signatures were never observed, the possible detection of these objects would represent a historical step toward understanding the composition and evolution of the Universe. Observations over the last two decades, however, have revealed that many multiply imaged quasars are microlensed by stars in lensing galaxies. In this volume, it is reviewed the state-of-art in computational microlensing. New advances in the area will have a big impact on our understanding of quasar and dark matter physics in the coming years. Time domain studies of lensed quasars are also promising tools to reveal the nature and structure of these distant sources. Modern dedicated or robotic 1-2 m class optical telescopes and sophisticated data reduction methods allow astronomers to obtain accurate light curves, and in some cases, to unambiguously detect intrinsic variations. Two independent contributions in this special issue show evidence supporting a reverberation/reprocessing scenario in lensed quasars at $1<z<3$.

I am grateful to the Editorial Board and Publishers of the "The Open Astronomy Journal" (www.bentham.org/open/toaaj), who allowed me to manage this volume and prepared an appropriate final version. I specially thank all contributors for their participation in the project. I also thank Gloria for her patience and understanding.

Luis J. Goicoechea

Departamento de Física Moderna

Universidad de Cantabria

Avda. de los Castros s/n

39005 Santander (Cantabria)

Spain

E-mail: goicol@unican.es

(C) Luis J. Goicoechea; Licensee Bentham Open.

This is an open access article licensed under the terms of the Creative Commons Attribution Non-Commercial License (http://creativecommons.org/ licenses/by-nc/3.0/) which permits unrestricted, non-commercial use, distribution and reproduction in any medium, provided the work is properly cited. 